\title{
Teoria das Molduras Relacionais e a tomada de perspectiva: Uma revisão dos protocolos de treinamento
}

\author{
Relational Frame Theory and perspective taking: A review of the training \\ protocols
}

Teoría de los Marcos Relacionales y toma de perspectivas: Revisión de los
protocolos de entrenamiento

Artur Lucchese Velozo', Leandro Azevedo Ribeiro', Márcio Santana da Silva' ${ }^{1}$

[1] Centro Universitário Nobre I Título abreviado: Molduras Relacionais e tomada de perspectiva I Endereço para correspondência: rua Inhanguape, $\mathrm{n}^{\circ}$ 138, Bairro Muchila I, Feira de Santana, Bahia, Brasil. Email: Artur Lucchese Velozo - artur.velozo@gmail.com I doi: 10.18761/PAC.2021.v12.RFT.11

Resumo: A tomada de perspectiva pode ser compreendida como a habilidade de interpretar, compreender e prever os comportamentos, pensamentos e emoções de si mesmo e dos outros, assumindo a perspectiva requisitada, sendo uma habilidade central no processo de socialização humana e déficits nessa capacidade estão associados a prejuízos nas habilidades sociais. Evidências apontam que o responder relacional arbitrariamente aplicável possui um papel significativo na tomada de perspectiva. O trabalho teve como objetivo analisar os protocolos de tomada de perspectiva baseados na RFT. Foram realizadas buscas em seis bases de dados, com 12 artigos selecionados para revisão. Foram registrados 4 protocolos diferentes, com duas abordagens teórico-conceituais divergentes. Os resultados são consistentes com os demais estudos, reforçando as evidências sobre a capacidade de aquisição dos repertórios por condicionamento operante, produção de respostas derivadas, e a existência de diferentes níveis de complexidade das molduras dêiticas, porém, os efeitos dos protocolos nas tarefas de teoria da mente ainda são inconsistentes. Por fim, algumas considerações sobre as atuais limitações e sobre o desenvolvimento da agenda de pesquisa do campo são sinalizadas.

Palavras-chave: molduras dêiticas, teoria das molduras relacionais, tomada de perspectiva, comportamento social. 
Abstract: Perspective-taking can be defined as the ability to interpret, understand and predict the behaviors, thoughts and emotions of oneself and others, assuming the required perspective. Perspective-taking is a central skill in the human socialization process, and deficits in this capacity are associated with impairments in social skills. Evidence points out that arbitrarily applicable relational responding plays a significant role in Perspective-taking. The objective of the work was to analyze the perspective-taking protocols based on the RFT. Searches were carried out in six databases, with 12 articles selected for review. Four different protocols were recorded, with two divergent theoretical-conceptual approaches. The results are consistent with the other studies, reinforcing the evidence on the ability to acquire the repertoires by operant conditioning, production of derived responses, and the existence of different levels of complexity of the deictic frames, however, the effects of the protocols on theory of mind tasks are still inconsistent. Finally, some considerations about the current limitations and the development of the field research agenda are signaled.

Keywords: deictic framing, relational frame theory, perspective-taking, social behavior.

Resumen: La toma de perspectiva se puede definir como la capacidad de interpretar, comprender y predecir las conductas, pensamientos y emociones de uno mismo y de los demás, asumiendo la perspectiva requerida, siendo una habilidad central en el proceso de socialización humana y los déficits en esta capacidad están asociados con deficiencias en las habilidades sociales. La evidencia señala que la respuesta relacional arbitrariamente aplicable juega un papel importante en la toma de perspectiva. El objetivo del trabajo fue analizar los protocolos de toma de perspectiva basados en el RFT. Las búsquedas se realizaron en seis bases de datos, con 12 artículos seleccionados para revisión. Se registraron cuatro protocolos diferentes, con dos enfoques teórico-conceptuales divergentes. Los resultados son consistentes con los otros estudios, reforzando la evidencia sobre la capacidad de adquirir los repertorios por condicionamiento operante, la producción de respuestas derivadas y la existencia de diferentes niveles de complejidad de los marcos deícticos, sin embargo, los efectos de los protocolos en las tareas de teoría de la mente siguen siendo inconsistentes. Finalmente, se señalan algunas consideraciones sobre las limitaciones actuales y el desarrollo de la agenda de investigación de campo.

Palabras clave: marcos dêiticos, teoría de los marcos relacionales, toma de perspectiva, comportamento social. 


\section{Teoria das Molduras Relacionais e a Tomada de Perspectiva: uma Revisão dos Protocolos de Treinamento.}

A Teoria das Molduras Relacionais ou RFT (Relational Frame Theory - Hayes et al., 2001) é uma explicação comportamental contemporânea da linguagem e da cognição, fornecendo um novo instrumental teórico para prever e influenciar comportamentos complexos. Entre as aplicações práticas da RFT encontram-se, por exemplo, a melhoria no desempenho em habilidades verbais e em medidas padronizadas de inteligência (e.g., Cassidy et al., 2010; Dixon et al., 2017), no funcionamento social (e.g., Vilardaga et al., 2012) e na tomada de perspectiva (e.g., Montoya-Rodriguez et al., 2017; Weil et al., 2011).

O conceito central da RFT é o operante denominado de Responder Relacional Arbitrariamente Aplicável (RRAA), que se refere a capacidade de responder relacionalmente a eventos ambientais, na qual a resposta não é controlada por aspectos formais ou físicos dos estímulos, mas sim pelas dicas contextuais fornecidas (Hayes et al., 2001; Rehfeldt et al., 2020). Sua aquisição ocorre através do treino de múltiplos exemplares com reforçamento diferencial do responder relacional não arbitrário, que é abstraído, ficando sob o controle de uma dica contextual, podendo então ser arbitrariamente aplicável. Um sujeito, por exemplo, sendo treinado para emitir respostas relacionais de comparação de tamanho, Maior/Menor, entre diferentes tipos de estímulos (carros, bolas, casas, pessoas, etc.), fica sob o controle da parcela específica desses exemplares que foi mantida constante, abstraindo essa propriedade relacional no contexto em que a dica Maior/Menor é apresentada. Após esse processo a resposta relacional se torna arbitrariamente aplicável a qualquer estímulo definido pelas relações convencionadas pela comunidade verbal (Rose \& Rabelo, 2012).

Tipos específicos de RRAA podem ser distinguidos pelo contexto antecedente (a dica relacional) que os evoca e são denominados molduras relacionais. Alguns tipos de molduras relacionais seriam: coordenação (A é igual a B), molduras causais (se
A, então B), comparação (A é melhor que B), oposição (A é oposto de B) e molduras dêiticas (Eu/ Você, Aqui/Ali, Agora/Depois) (Hayes et al., 2001).

A RFT compreende a tomada de perspectiva como uma habilidade complexa, composta pelos diferentes repertórios que envolvem molduras (respostas relacionais) dêiticas. Essa habilidade pode ser compreendida como as capacidades de interpretar, compreender e prever os comportamentos, pensamentos e emoções de si mesmo e dos outros, assumindo a perspectiva requisitada (Kavanagh et al., 2020). Os repertórios envolvidos seriam os três tipos de molduras dêiticas relacionais (MDR): pessoal (Eu/Você), espacial (Aqui/Ali) e temporal (Agora/Depois). Estas molduras seriam estabelecidas através de diversas perguntas (treino de múltiplos exemplares) como "O que eu estou fazendo aqui??, “Onde você está agora?”, “O que você estava fazendo depois?", variando elementos ambientais, porém, mantendo constante as propriedades relacionais especificas de cada MDR (McHugh, 2015)

Em McHugh et al. (2004) houve uma das primeiras tentativas de operacionalizar tarefas de MDR no formato de um protocolo de avaliação e aplicação (Barnes-Holmes Perspective-Taking Protocol; BH-PTP), para mensurar o desempenho de populações típicas de crianças e adultos. Foi elaborado um protocolo composto por 62 itens, contendo os três tipos de MDR, organizados em três níveis de complexidade. No nível simples, a resposta relacional não exigia nenhuma inversão, "Eu estou segurando uma pedra preta, você está segurando uma pedra azul. Qual pedra você estásegurando? Qual pedra eu estou segurando?"; já no nível reverso, uma relação é invertida na pergunta, "Eu estou sentado aqui na cadeira rosa, você está sentado ai na cadeira verde. Se eu fosse você e você fosse eu, onde eu estaria sentado? E você?". Por fim, no nível duplo-reverso, as duas relações são invertidas: "Eu estou sentado aqui na cadeira rosa, você está sentado ali na cadeira verde. Se eu fosse você e você fosse eu, e aqui fosse ali e ali fosse aqui, onde eu estaria sentado? E você?".

Através do BH-PTP os repertórios de MDR já foram estabelecidos em crianças típicas (Davlin et al., 2011; Weil et al., 2011) e atípicas, crianças diagnosticadas com autismo (Gilroy, et al., 2015); já em populações adultas foram identificados déficits nas 
MDR em sujeitos com anedonia social (Vilardaga et al., 2012; Villatte et al., 2008), ansiedade social (Janssen et al., 2014) e esquizofrenia (Villatte et al., 2010 a, 2010b). Essas evidências sinalizam que a RFT apresenta a capacidade de gerar protocolos de treinamento que podem contribuir para a melhoria dos prejuízos registrados nestas populações (Montoya-Rodriguez et al., 2017).

A elaboração do BH-PTP permitiu uma abordagem inteiramente nova para a tomada de perspectiva, cuja parte majoritária das investigações ocorriam sob o prisma da psicologia cognitiva, através do conceito de Teoria da Mente (ToM). Esta pode ser conceituada como a habilidade de explicar e prever comportamentos através de atribuição de estados mentais a outros e a si mesmo (Kavanagh et al., 2020); uma tarefa tradicional de ToM pode envolver uma série de imagens da região dos olhos de faces humanas e os participantes devem indicar pensamentos ou emoções associadas aos sujeitos das imagens (Baron-Cohen et al., 2001), ou, com a apresentação de diferentes histórias, solicitando o participante a responder sobre as crenças ou falsas-crenças dos personagens envolvidos (Hendriks et al., 2016).

A variação do desempenho no $\mathrm{BH}-\mathrm{PTP}$ ao longo das faixas etárias são convergentes com a literatura sobre o desenvolvimento das habilidades em ToM (McHugh et al., 2004) e existem evidências de que a aquisição dos repertórios de MDR está correlacionada a melhores desempenhos em tarefas de ToM (O’Neil \& Weil, 2014; Villatte, et al., 2008; Weil et al., 2011).

Em Montoya-Rodriguez et al. (2017) foi realizada a primeira revisão de literatura orientada especificamente para as MDR, incluindo toda a produção empírica e teórica da área entre os anos de 2001 e 2015. Os autores encontraram que quase a totalidade das pesquisas empíricas utilizaram amostras de populações típicas, com a maioria de crianças. Os desenhos de estudo mais frequente foram: populações adultas típicas com desenhos não manipulativos, e crianças típicas com desenhos manipulativos. Mais de 70\% dos artigos tiveram como foco temático as relações com a ToM.

Com o intuito de prosseguir com a revisão de Montoya-Rodriguez et al. (2017) em relação somente à produção de artigos empíricos, o presente trabalho revisou as pesquisas primárias que utilizaram a RFT para investigar a tomada de perspectiva, publicados em periódicos entre o período de 2016 a 2020

O objetivo dessa revisão foi analisar os protocolos de tomada de perspectiva baseados na RFT por meio: (1) da caracterização dos protocolos de MDR, (2) da descrição dos desempenhos nos protocolos, (3) do exame das relações entre os desempenhos nos protocolos e nas demais tarefas de tomada de perspectiva (e.g. ToM).

\section{Método}

Essa investigação é uma revisão de literatura de abordagem integrativa, com o intuito de revisar as evidências para elaborar uma compreensão ampliada do fenômeno através de uma articulação coerente e tangível (Souza et al., 2010).

Os estudos foram obtidos através de uma busca nas bases de dados PubMed, Google Scholar, EBSCOhost, Portal de Periódicos da CAPES, Biblioteca Virtual de Saúde e SciELO, na forma de artigos, publicados entre 2016 e 2020 em inglês, português e espanhol. As buscas nos três idiomas selecionados foram realizadas através do mesmo procedimento, com a adequação dos descritores. Foram utilizadas as palavras-chave "Molduras Dêiticas", "Respostas Dêiticas", "Responder Relacional Dêitico" e "Teoria das Molduras Relacionais" cada uma individualmente utilizando o operador "AND" com a palavra-chave "Tomada de Perspectiva", para os artigos em português; "Relaciones deícticas", "Marcos Deícticos", “Teoría de los Marcos Relacionales", "Marcos Relacionales", com o operador "AND" e "Toma de Perspectiva", para os artigos em espanhol; "Deictic Framing", "Deictic Relations", "Relational Frame Theory", "Relational frames", operador "AND" e "Perspective Taking" para os artigos em inglês. Foi utilizado como parâmetro a presença das palavras-chaves em qualquer parte do trabalho (resumo, título ou assunto). Os resultados da busca foram organizados por data, iniciando pelo mais recente.

Foram selecionadas investigações, através da leitura dos resumos que atenderam aos seguintes critérios: 1) utilizar a teoria das molduras relacio- 


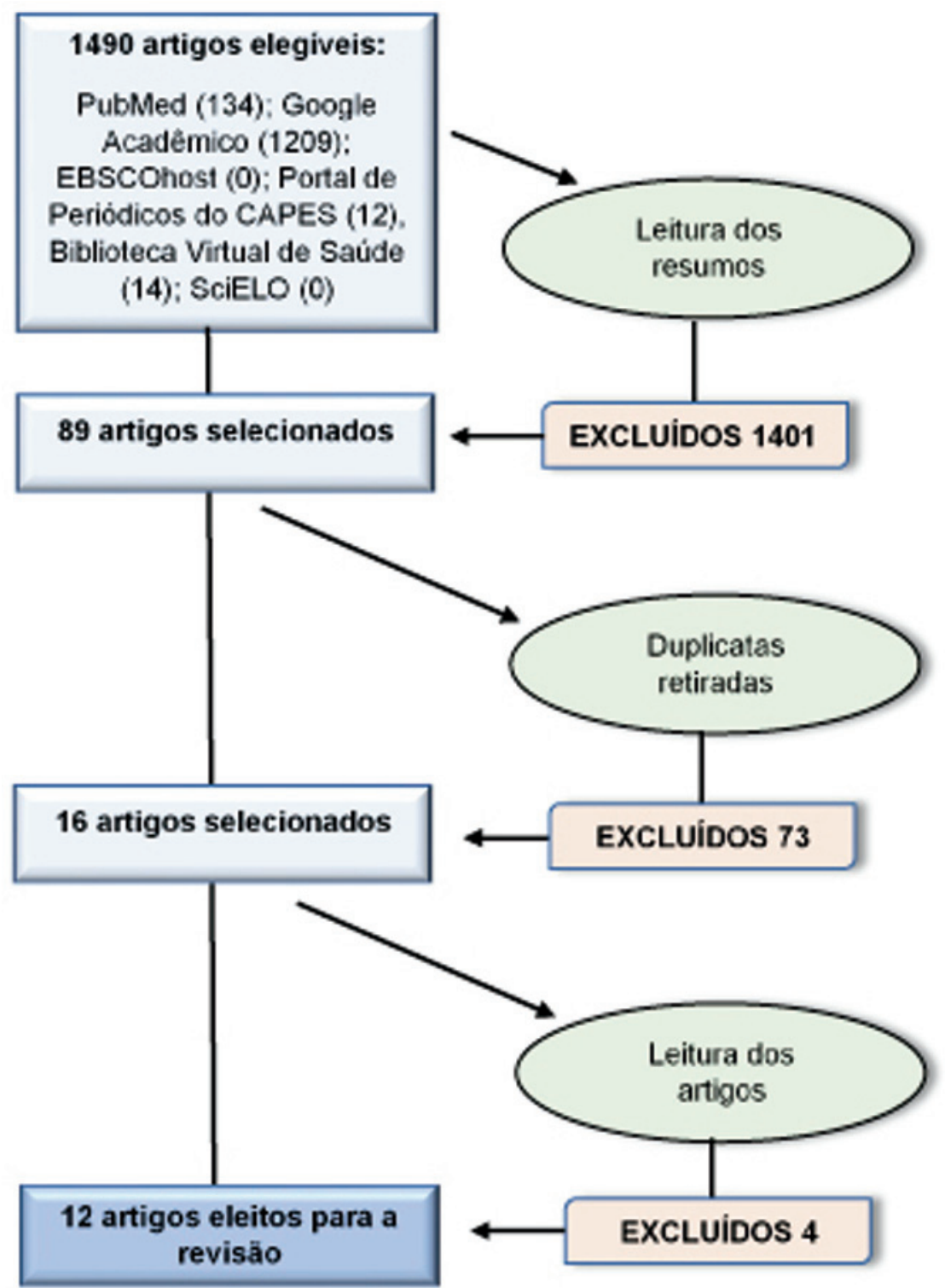

Figura 1. Percurso metodológico da seleção dos artigos revisados

nais como fundamentação teórica, 2) investigar as MDR, 3) ser um estudo primário, 4) escrito em português, inglês ou espanhol.

Foram excluídos artigos que: 1) os objetivos do estudo não envolviam diretamente o RRAA na tomada de perspectiva; 2) não operacionalizavam as MDR exclusivamente sob a perspectiva da RFT; 3) não estavam publicados em periódicos científicos.

Cada estudo foi classificado como uma unidade de registro, as quais foram posteriormente agrupadas em unidades de contexto, em função do tipo de protocolo utilizado no artigo. O instrumento para coleta de dados indicado por Souza et al. (2010) foi utilizado para orientar a extração e codificação dos dados das unidades de registro, com as seguintes adições: (1) em "Intervenções utilizadas", foi especificado o protocolo dêitico utilizado; (2) em "Resultados", foi especificado o desempenho nos protocolos dêiticos, na tomada de perspectiva e a derivação das respostas.

As seguintes categorias utilizadas por Montoyta-Rodriguez et al. (2017) também foram aplicadas na atual revisão: estudos manipulativos, não manipulativos, populações típicas e atípicas, crianças pequenas, crianças grandes e adultos. Pela 
inclusão somente de artigos empíricos as demais categorias não foram utilizadas.

Seguindo os critérios estabelecidos por Montoyta-Rodriguez et al. (2017) para a inclusão nas categorias, os estudos que sistematicamente alteraram variáveis para modificar o comportamento dos participantes, foram classificados possuindo o desenho manipulativo. Estudos que registraram dados de pelo menos uma variável sem a manipulação, foram enquadrados como não manipulativos. Estudos cujos participantes não apresentavam diagnóstico clínico foram classificados como população típica; estudos com participantes que possuíam diagnósticos clínicos foram classificados como atípicos. Nos casos em que a amostra era composta por ambos, as duas classificações foram atribuídas. Amostras constituídas por participantes na faixa etária até 7 anos foram enquadradas como crianças pequenas; de 8 a 17 anos como crianças grandes; 18 anos ou mais como adultos.

Com o intuito de melhor explorar os resultados dos artigos empíricos, alinhado com os objetivos de avaliar o desempenho nos protocolos e nas tarefas tradicionais de tomada de perspectiva, além das categorias de Montoyta-Rodriguez et al. (2017) foram incluídas na presente revisão categorias associadas aos resultados dos procedimentos aplicados, expostas a seguir: (1) características dos protocolos de MDR utilizados; (2) efeitos dos protocolos de treinamento nos repertórios de MDR dos participantes; (3) efeitos dos protocolos nas demais tarefas de tomada de perspectiva e (4) derivação das respostas treinadas pelo protocolo para estímulos não treinados.

As etapas de triagem dos artigos, extração dos dados e categorização foram realizadas somente por um dos autores.

\section{Resultados}

Foram registrados 1.490 artigos após a aplicação dos descritores, operadores e filtros; com a leitura dos resumos, 85 artigos foram selecionados, 73 eram duplicatas que foram retiradas, restando 12 artigos que foram escolhidos para a revisão.

$\mathrm{Na}$ Tabela 1, constam as categorias aplicadas a cada artigo revisado, com os principais resultados de cada investigação.
Foram registrados 5 estudos utilizando o BH-PTP, 3 aplicando o IRAP (Implicit Relational Assessment Procedure), 2 artigos com o PEAK-T (Promoting the Emergence of Advanced Knowledge - Transformation) e 2 artigos com o RT-PTP (Relational Triangulation Perspective-Taking Protocol).

Dos artigos revisados, 4 (33\%) foram classificados como manipulativos e 8 (67\%) como não manipulativos. Em relação à população investigada, foram registrados 9 (59\%) artigos utilizando populações típicas (6 - 40\% - com adultos, 1 com crianças grandes e 2 com crianças pequenas). Já em relação às populações atípicas, foram identificados $6(40 \%)$ artigos (4 - 26\% - com adultos, 1 com crianças pequenas e $1 \mathrm{com}$ crianças grandes $)^{1}$.

Os protocolos BH-PTP e o PEAK-T são medidas explícitas nas quais os resultados são mensurados a partir do número de tentativas respondidas corretamente no protocolo, e os repertórios são identificados como estabelecidos quando os participantes atingem o critério de $80 \%$ a $100 \%$ de acertos. Já o IRAP é uma medida implícita, baseada em latência, que mede a força das respostas relacionais (ou viés) comparando o tempo de resposta à diferentes tipos de relações entre estímulos. Por fim, no RT-PTP também são mensurados o número de acertos dos participantes, mas a presença do repertório é registrada quando determinado estímulo arbitrário adquire controle contextual espontâneo sobre a derivação de respostas; também uma medida explícita, mas de características distintas do BH-PTP e PEAK-T.

Quatro estudos somente mensuraram os desempenhos dos participantes, ou seja, não realizaram treinamento e aquisição de MDR (BarberoRubio et al., 2016; Hendriks et al., 2016; Kavanagh et al., 2018, 2019).

1 Porcentagens das populações estão baseadas no total de 15 , maior que a amostra de artigos revisados, pois mais de um tipo de população pode estar presente no mesmo estudo alterando o valor total. 
Tabela 1. Artigos revisados com suas respectivas categorias descritas.

\begin{tabular}{|c|c|c|c|c|c|}
\hline Autores & Desenho & $\begin{array}{l}\text { População e } \\
\text { Amostra (N) }\end{array}$ & Objetivos & Protocolo & Principais Resultados \\
\hline $\begin{array}{l}\text { Arango \& } \\
\text { Baquero } \\
(2017)\end{array}$ & $\begin{array}{l}\text { Não } \\
\text { Manipulativo }\end{array}$ & $\begin{array}{l}\text { Crianças pequena } \\
\text { e grande, típicas. } \\
\mathrm{N}=2 \\
\text { (7 e } 8 \text { anos) }\end{array}$ & $\begin{array}{l}\text { Analisar a aquisição } \\
\text { de repertório de } \\
\text { respostas relacionais } \\
\text { dêiticas pessoais, es- } \\
\text { paciais e temporais. }\end{array}$ & $\begin{array}{l}\text { BH-PTP } \\
\text { (Explicito) }\end{array}$ & $\begin{array}{l}\text { Os repertórios do nível simples já esta- } \\
\text { vam estabelecidos em um participante. } \\
\text { Participantes atingiram } 100 \% \text { de } \\
\text { acertos no nível reverso, porém seu } \\
\text { desempenho foi reduzido na ausência } \\
\text { de reforço e ajuda. } \\
\text { Participantes não atingiram o critério de } \\
\text { maestria no nível reverso e não progre- } \\
\text { diram para o nível duplo-reverso. } \\
\text { Todas as MDR no nível simples registra- } \\
\text { ram respostas derivadas, assim como, } \\
\text { no nível reverso, porém com variações } \\
\text { entre os participantes. }\end{array}$ \\
\hline $\begin{array}{l}\text { Hendriks et al. } \\
\text { (2016) }\end{array}$ & $\begin{array}{l}\text { Não manipu- } \\
\text { lativo } \\
\text { Sem C.O. }{ }^{1}\end{array}$ & $\begin{array}{l}\text { Adultos, atípicos e } \\
\text { típicos. } \\
\mathrm{N}=58 \\
(18-54 \text { anos })\end{array}$ & $\begin{array}{l}\text { Investigar a relação } \\
\text { do protocolo com } \\
\text { duas medidas de } \\
\text { ToM (Faux-pas test e } \\
\text { Strange } \\
\text { Stories test). }\end{array}$ & $\begin{array}{l}\text { BH-PTP } \\
\text { (Explícito) }\end{array}$ & $\begin{array}{l}\text { Correlação positiva significativa entre o } \\
\text { Faux-pas test com as MDR pessoais e } \\
\text { com o nível reverso. } \\
\text { Padrão similiar para o Strange } \\
\text { Stories test, mas sem significância após } \\
\text { correção para inteligência. }\end{array}$ \\
\hline $\begin{array}{l}\text { Montoya- } \\
\text { Rodriguez \& } \\
\text { Molina-Cobos } \\
\text { (2016) }\end{array}$ & Manipulativo & $\begin{array}{l}\text { Crianças peque- } \\
\text { nas, típicas. } \\
\text { N Experimental = } \\
3 \text { ( } 5 \text { anos) } \\
\text { N Controle = } 3 \\
\text { (5 anos) }\end{array}$ & $\begin{array}{l}\text { Examinar uma versão } \\
\text { modificada do pro- } \\
\text { tocolo, com menos } \\
\text { itens e com tentativas } \\
\text { interativas. }\end{array}$ & $\begin{array}{l}\text { BH-PTP } \\
\text { (Explícito) }\end{array}$ & $\begin{array}{l}\text { O nível simples já estava estabelecido } \\
\text { nos participantes. } \\
\text { Ocorreu a aquisição dos repertórios de } \\
\text { MDR pessoais, espaciais e temporais } \\
\text { através de uma história de reforçamen- } \\
\text { to. } \\
\text { Foi necessário treino adicional para } \\
\text { estabelecer a distinção entre o nível } \\
\text { reverso e duplo-reverso. } \\
\text { Participantes experimentais registraram } \\
\text { derivação de respostas registrado no } \\
\text { pós-teste. } \\
\text { Não foram registrados efeitos do treino } \\
\text { nas tarefas de ToM. }\end{array}$ \\
\hline $\begin{array}{l}\text { Montoya- } \\
\text { Rodriguez } \\
\& \text { McHugh } \\
(2017)\end{array}$ & $\begin{array}{l}\text { Não } \\
\text { Manipulativo }\end{array}$ & $\begin{array}{l}\text { Adulto, atípico. } \\
\text { N=1 (24 anos) }\end{array}$ & $\begin{array}{l}\text { Treinar as MDR } \\
\text { em um adulto com } \\
\text { Síndrome de Down. }\end{array}$ & $\begin{array}{l}\text { BH-PTP } \\
\text { (Explícito) }\end{array}$ & $\begin{array}{l}\text { Participante demonstrou domínio das } \\
\text { relações simples na linha de base. } \\
\text { Somente as MDR reversas foram treina- } \\
\text { das. } \\
\text { O participante obteve domínio nas } \\
\text { relações reversas pessoais e espaciais } \\
\text { com uma sessão de treino, e com duas } \\
\text { sessões de treino para as relações } \\
\text { temporais. } \\
\text { Déficits nas MDR reversas foram reme- } \\
\text { diados pelo treino. }\end{array}$ \\
\hline
\end{tabular}




\begin{tabular}{|c|c|c|c|c|c|}
\hline Autores & Desenho & $\begin{array}{l}\text { População e } \\
\text { Amostra (N) }\end{array}$ & Objetivos & Protocolo & Principais Resultados \\
\hline $\begin{array}{l}\text { Montoya- } \\
\text { Rodriguez \& } \\
\text { Molina-Cobos } \\
\text { (2019) }\end{array}$ & Manipulativo & $\begin{array}{l}\text { Adultos, atípicos. } \\
\text { N Experimental }= \\
4 \text { (29-34 anos) } \\
\text { N Controle }=4 \\
(18-28 \text { anos })\end{array}$ & $\begin{array}{l}\text { Adaptar o BH-PTP } \\
\text { para as tarefas de } \\
\text { ToM e avaliar o } \\
\text { impacto do protocolo } \\
\text { nas tarefas de ToM e } \\
\text { nas MDR, em adultos } \\
\text { com Síndrome de } \\
\text { Down. }\end{array}$ & $\begin{array}{l}\text { BH-PTP } \\
\text { (Explícito) }\end{array}$ & $\begin{array}{l}\text { As relações simples já estavam estabe- } \\
\text { lecidas na linha de base. } \\
\text { O treinamento melhorou o desempenho } \\
\text { no protocolo modificado, nos níveis } \\
\text { reverso e duplo-reverso. } \\
\text { Não houveram alterações no } \\
\text { desempenho do BH-PTP tradicional. } \\
\text { Todos os participantes experimentais } \\
\text { registraram melhorias no desempenho } \\
\text { nas tarefas de ToM. }\end{array}$ \\
\hline $\begin{array}{l}\text { Barbero-Rubio } \\
\text { et al. (2016) }\end{array}$ & $\begin{array}{l}\text { Não manipu- } \\
\text { lativo } \\
\text { Sem C.O. }{ }^{1}\end{array}$ & $\begin{array}{l}\text { Adultos, típicos. } \\
\begin{array}{l}\mathrm{N}=35 \\
\text { (18-38 anos) }\end{array}\end{array}$ & $\begin{array}{l}\text { Utilizar o IRAP para } \\
\text { mensurar a comple- } \\
\text { xidade e flexibilidade } \\
\text { na tomada de pers- } \\
\text { pectiva, e relacionar } \\
\text { o IRAP com medidas } \\
\text { explícitas (BH-PTP e } \\
\text { Perspective-Taking } \\
\text { Scale). }\end{array}$ & $\begin{array}{l}\text { IRAP } \\
\text { (Implícito) }\end{array}$ & $\begin{array}{l}\text { Respostas afirmativas sobre sua própria } \\
\text { perspectiva registraram menor tempo } \\
\text { de reação, maior viés, do que respostas } \\
\text { sobre a perspectiva do outro. } \\
\text { Valores elevados do Dirap indicaram } \\
\text { baixa flexibilidade. } \\
\text { IRAP foi sensível para registrar os } \\
\text { diferentes níveis de complexidade e a } \\
\text { flexibilidade relacional. } \\
\text { Diferenças significativas entre os } \\
\text { acertos nas tentativas reversas e duplo- } \\
\text {-reversas do BH-PTP. } \\
\text { Tentativas duplo-reversas correlaciona- } \\
\text { das negativamente com o valor Dirap. } \\
\text { Ausência de correlações significativas } \\
\text { nos protocolos dêiticos e a Perspective- } \\
\text { Taking Scale. }\end{array}$ \\
\hline $\begin{array}{l}\text { Kavanagh et al. } \\
\text { (2018) }\end{array}$ & $\begin{array}{l}\text { Manipulativo } \\
\text { Sem C.O. }{ }^{1}\end{array}$ & $\begin{array}{l}\text { Adultos, típicos. } \\
\text { N1=40 } \\
\text { (18-36 anos) } \\
\text { N2=66 } \\
\text { (18-48 anos) }\end{array}$ & $\begin{array}{l}\text { Mensurar a flexibili- } \\
\text { dade e complexida- } \\
\text { de, e comparar os } \\
\text { desempenhos entre } \\
\text { um IRAP (Dêitico) } \\
\text { que requisita a } \\
\text { perspectiva do par- } \\
\text { ticipante, com um } \\
\text { IRAP (Controle) que } \\
\text { requisita somente } \\
\text { a perspectiva de } \\
\text { outros. }\end{array}$ & $\begin{array}{l}\text { IRAP } \\
\text { (Implícito) }\end{array}$ & $\begin{array}{l}\text { Ambos tipos de IRAP utilizados regis- } \\
\text { traram maior viés para tentativas que } \\
\text { envolviam molduras de coordenação. } \\
\text { IRAP Dêitico produziu um efeito de } \\
\text { dominância de um tipo de tentativa } \\
\text { (coordenação sobre sua própria pers- } \\
\text { pectiva). } \\
\text { IRAP Controle produziu um efeito de } \\
\text { dominância de dois tipos de tentativas } \\
\text { (ambas tentativas que envolviam moldu- } \\
\text { ras de coordenação). } \\
\text { A adoção do procedimento de leitura } \\
\text { em voz alta das tentativas pode ter } \\
\text { atenuado o efeito diferencial de tipo de } \\
\text { tentativa. }\end{array}$ \\
\hline
\end{tabular}




\begin{tabular}{|c|c|c|c|c|c|}
\hline Autores & Desenho & $\begin{array}{l}\text { População e } \\
\text { Amostra (N) }\end{array}$ & Objetivos & Protocolo & Principais Resultados \\
\hline $\begin{array}{l}\text { Kavanagh et al. } \\
\text { (2019) }\end{array}$ & $\begin{array}{l}\text { Manipulativo. } \\
\text { Sem C.O. }{ }^{1}\end{array}$ & $\begin{array}{l}\text { Adultos }^{2} \text {, típicos. } \\
\text { N1=55 } \\
(17-38 \text { anos }) \\
\text { N2 }=51 \\
\text { (18-49 anos) } \\
\text { N3=40 } \\
(18-45 \text { anos }) \\
\text { N4=34 } \\
(18-39 \text { anos }) \\
\text { N5=32 } \\
(18-32 \text { anos }) \\
\text { N6=34 } \\
(18-50 \text { anos })\end{array}$ & $\begin{array}{l}\text { Avaliar o responder } \\
\text { relacional dêitico no } \\
\text { que se refere aos } \\
\text { estados mentais dos } \\
\text { outros (IRAP-Outro) } \\
\text { e de si mesmo } \\
\text { (IRAP-Eu). } \\
\text { Experimentos } 1 \text { e } 2 \\
\text { utilizaram o outro não } \\
\text { especificado. } \\
\text { Experimentos } 3 \text { a } 6 \\
\text { especificaram o outro } \\
\text { através de diversos } \\
\text { aspectos (ex: similari- } \\
\text { dade e diferença). }\end{array}$ & $\begin{array}{l}\text { IRAP } \\
\text { (Implícito) }\end{array}$ & $\begin{array}{l}\text { Viés significativamente maior para res- } \\
\text { ponder sobre os estados mentais dos } \\
\text { outros, quando o outro não é especifi- } \\
\text { cado. } \\
\text { Ao especificar o outro, não foram regis- } \\
\text { tradas diferenças significativas entre os } \\
\text { tipos de IRAP. } \\
\text { Os efeitos IRAP registrados foram na } \\
\text { direção predita pelo modelo da história } \\
\text { pré-experimental de reforçamento. }\end{array}$ \\
\hline $\begin{array}{l}\text { Belisle et al. } \\
(2016)\end{array}$ & $\begin{array}{l}\text { Não manipu- } \\
\text { lativo }\end{array}$ & $\begin{array}{l}\text { Crianças grandes } \\
\text { e adulto, atípicos. } \\
\begin{array}{l}\mathrm{N}=3 \\
(12,14 \text { e } 18 \text { anos })\end{array}\end{array}$ & $\begin{array}{l}\text { Avaliar a eficácia } \\
\text { de um protocolo de } \\
\text { treinamento de MDR } \\
\text { pessoais reversas } \\
\text { para crianças com } \\
\text { autismo. }\end{array}$ & $\begin{array}{l}\text { PEAK-T } \\
\text { (Explícito) }\end{array}$ & $\begin{array}{l}\text { Os participantes não demonstraram os } \\
\text { repertórios de MDR pessoais reversas } \\
\text { na linha de base. } \\
\text { Os participantes adquiriram o repertório } \\
\text { de MDR após o treino pelo PEAK-T. } \\
\text { Dois participantes demonstraram deri- } \\
\text { vação de MDR pessoais reversas "Eu" } \\
\text { sem treino direto. } \\
\text { Todos participantes demonstraram } \\
\text { transformação de função de estímulo } \\
\text { discriminativo. }\end{array}$ \\
\hline $\begin{array}{l}\text { Barron et al. } \\
(2018)\end{array}$ & $\begin{array}{l}\text { Não } \\
\text { Manipulativo }\end{array}$ & $\begin{array}{l}\text { Crianças peque- } \\
\text { nas, atípicas. } \\
\mathrm{N}=2 \text { (6 anos) }\end{array}$ & $\begin{array}{l}\text { Avaliar a efetividade } \\
\text { do treinamento de } \\
\text { MDR espaciais e } \\
\text { temporais reversas } \\
\text { para crianças com } \\
\text { autismo. }\end{array}$ & $\begin{array}{l}\text { PEAK-T } \\
\text { (Explícito) }\end{array}$ & $\begin{array}{l}\text { Os participantes não demonstraram os } \\
\text { repertórios de MDR reversas. } \\
\text { Participantes foram capazes de adquirir } \\
\text { os repertórios após o treino pelo } \\
\text { PEAK-T. } \\
\text { Participantes foram capazes de derivar } \\
\text { respostas por implicação mútua. } \\
\text { Os repertórios estabelecidos apre- } \\
\text { sentaram transformação de função de } \\
\text { estímulo discriminativo. }\end{array}$ \\
\hline $\begin{array}{l}\text { Guinther } \\
(2017)\end{array}$ & $\begin{array}{l}\text { Não manipu- } \\
\text { lativo }\end{array}$ & $\begin{array}{l}\text { Adultos, típicos. } \\
\text { N=8 (23-29 anos) }\end{array}$ & $\begin{array}{l}\text { Demonstrar o contro- } \\
\text { le contextual sobre } \\
\text { a derivação das } \\
\text { perspectivas mate- } \\
\text { riais entre o outro e o } \\
\text { estímulo. }\end{array}$ & $\begin{array}{l}\text { RT-PTP } \\
\text { (M1 -Módulo 1: } \\
\text { Crenças } \\
\text { Verdadeiras) } \\
\text { (Explícito) }\end{array}$ & $\begin{array}{l}\text { Foi estabelecido o controle contextual } \\
\text { sobre a triangulação da tomada de } \\
\text { perspectiva, quando participantes foram } \\
\text { requisitados para responder de acordo } \\
\text { com as crenças verdadeiras de outros. } \\
\text { Sob o controle dos estímulos contex- } \\
\text { tuais } \\
\text { os participantes demonstram derivação } \\
\text { espontânea que os outros se comporta- } \\
\text { riam da mesma forma que eles. } \\
\text { A transformação de função de estímulo } \\
\text { não foi conspícua, pois os alinhamentos } \\
\text { interpessoais foram de coordenação. }\end{array}$ \\
\hline
\end{tabular}




\begin{tabular}{|c|c|c|c|c|c|}
\hline Autores & Desenho & $\begin{array}{l}\text { População e } \\
\text { Amostra (N) }\end{array}$ & Objetivos & Protocolo & Principais Resultados \\
\hline $\begin{array}{l}\text { Guinther } \\
\text { (2018) }\end{array}$ & $\begin{array}{l}\text { Não } \\
\text { Manipulativo }\end{array}$ & $\begin{array}{l}\text { Adultos, típicos. } \\
\mathrm{N}=4 \text { (19-21 anos) }\end{array}$ & $\begin{array}{l}\text { Estabelecer o con- } \\
\text { trole contextual sobre } \\
\text { falsas-crenças e } \\
\text { triangular a deriva- } \\
\text { ção de respostas } \\
\text { de falsas-crenças } \\
\text { materiais em outros. }\end{array}$ & $\begin{array}{l}\text { RT-PTP } \\
\text { (M2 -Módulo 2: } \\
\text { Falsas } \\
\text { Crenças) } \\
\text { (Explícito) }\end{array}$ & $\begin{array}{l}\text { Replicou os dados de Guinter (2017), } \\
\text { com a triangulação das crenças-verda- } \\
\text { deiras materiais de outros e derivação } \\
\text { de respostas. } \\
\text { Para dois participantes o RT-PTP-M1 } \\
\text { foi o suficiente para estabelecer o } \\
\text { controle contextual de estímulos para a } \\
\text { transposição espontânea do repertório } \\
\text { pré-experimental de falsas-crenças. } \\
\text { Foi estabelecido um novo repertório de } \\
\text { respostas de falsas-crenças. } \\
\text { RT-PTP-M2 estabeleceu o controle } \\
\text { contextual sobre a derivação espon- } \\
\text { tânea da perspectiva do Outro1 para } \\
\text { o Outro2, através do processo de } \\
\text { triangulação relacional, por meio da co- } \\
\text { ordenação do alinhamento interpessoal } \\
\text { Outro-Outro. } \\
\text { O repertório de respostas de falsas- } \\
\text {-crenças foi derivado. }\end{array}$ \\
\hline
\end{tabular}

Nota. 1 C.O. = Condicionamento Operante.

2 População foi classificada somente como adulta, apesar da presença de participantes com 17 anos, pela composição expressivamente majoritária de adultos nas amostras.

\section{Discussão}

Foram registrados artigos predominantemente com desenhos do tipo não manipulativo, e somente 2 artigos com desenhos do tipo manipulativo realizaram treino das MDR (Montoya-Rodriguez \& MolinaCobos, 2016, 2019), significando que poucos dados foram produzidos sobre os impactos dos treinos das MDR com a manipulação de variáveis independentes. Isto sinaliza a persistência de uma lacuna nos estudos manipulativos, também apontada por Montoya-Rodriguez et al. (2017), dificultando uma agenda de previsão e influência do comportamento.

As populações com menos pesquisas foram crianças pequenas atípicas e adultos atípicos no trabalho de Montoya-Rodriguez et al. (2017). Foi possível registrar que os estudos com crianças pequenas atípicas permaneceram com poucas investigações e números amostrais reduzidos (Barron et al., 2018); já para adultos atípicos houve um crescimento relativo nas pesquisas, (Belisle et al., 2016; Hendriks et al., 2016; Montoya-Rodriguez \& McHugh, 2017; Montoya-Rodriguez \& Molina-Cobos, 2019), mas apenas um desses artigos registrou um número amostral maior do que 8 (Hendriks et al, 2016), indicando que a generalidade de tais resultados devem ser encarados com cautela e que são necessários trabalhos mais robustos.

Todos os artigos revisados demonstraram que os repertórios investigados podem ser estabelecidos através de uma história de reforçamento, em consonância às evidências previamente registradas. Isto aponta que a tomada de perspectiva é composta por habilidades que respondem e podem ser modeladas por contingências de reforçamento, fornecendo uma base sólida para o desenvolvimento de procedimentos e protocolos que podem remediar déficits e aprimorar os desempenhos das populações que apresentam comprometimentos nessas habilidades.

Em virtude das similaridades conceituais e metodológicas, os resultados dos protocolos $\mathrm{BH}$ PTP e PEAK-T serão discutidos conjuntamente. Os resultados indicam que os repertórios do nível simples das MDR estão estabelecidos na maioria 
das populações clínicas e não-clínicas (Arango \& Baquero, 2017; Hendriks et al., 2016; MontoyaRodriguez \& Molina-Cobos, 2016; MontoyaRodríguez \& McHugh, 2017; Montoya-Rodriguez \& Molina-Cobos, 2019), com a produção de respostas derivadas (Arango \& Baquero, 2017). Já os repertórios envolvidos no nível reverso não aparecem estabelecidos e, portanto, o desempenho das populações costuma ser menor nesse nível, elevando o número de acertos com o treinamento das respostas relacionais dêiticas (Arango \& Baquero, 2017; Barron et al., 2018; Belisle et al., 2016 ; Montoya-Rodriguez \& Molina-Cobos, 2016; Montoya-Rodríguez \& McHugh, 2017), produzindo derivação das respostas treinadas (Barron et al., 2018; Belisle et al., 2016).

Levando em consideração o nível reverso, há evidências de que as relações temporais necessitam de uma história mais longa de reforçamento para se estabelecer, relativo às molduras espaciais e pessoais (Arango \& Baquero, 2017; Montoya-Rodriguez \& Molina-Cobos, 2016). Por fim, o nível duplo-reverso é significativamente diferente do nível reverso e aparenta ser um indicador de flexibilidade na mudança de perspectiva (Barbero-Rubio et al., 2016). Esses resultados reforçam a compreensão da existência de diferentes níveis de complexidade das MDR (McHugh et al., 2004).

Os resultados obtidos através do IRAP sinalizam um maior viés para responder sob o controle de molduras de coordenação do que oposição (Barbero-Rubio et al., 2016; Kavanagh et al., 2018, 2019), sendo o viés maior para respostas que envolvem a coordenação e a perspectiva do participante, "Eu" (Barbero-Rubio et al., 2016; Kavanagh et al., 2018). Os resultados podem explicados pelo modelo differential arbitrarily applicable relational responding effects (DAARRE), onde as repostas relacionais com uma maior história de reforçamento apresentam máxima coerência e tempos de reação menores (Kavanagh et al., 2018). Desta forma, o IRAP foi sensível para a flexibilidade e complexidade das MDR (Barbero-Rubio et al., 2016; Kavanagh et al., $2018,2019)$. O IRAP parece ser sensível também aos diferentes tipos de procedimentos adotados na execução do protocolo, alterando significativamente os desempenhos dos participantes, como a especificação do outro nas tentativas, reduzindo o viés para responder sobre sua própria perspectiva (Kavanagh et al., 2019), ou a adição de leitura em voz alta pelos participantes (Kavanagh et al., 2018), reduzindo os efeitos diferenciais dos tipos de tentativas.

Porém, vale ressaltar que em Kavanagh et al. (2019) o IRAP não foi sensível às diferenças entre a perspectiva do participante e do outro, com exceção de um tipo especifico de tentativa, o que difere de duas outras investigações (Barbero-Rubio et al., 2016; Kavanagh et al., 2018).

Quanto às correlações do desempenho nas MDR e nas tarefas de ToM, Montoya-Rodriguez e MolinaCobos (2016) não registraram efeitos do protocolo nas tarefas de ToM e Hendriks et al. (2016) registraram melhorias somente em uma de duas tarefas de ToM, com correlação significativa entre as relações reversas e o desempenho nas tarefas em ToM. Por fim, Montoya-Rodriguez e Molina-Cobos (2019) registraram melhorias significativas nas tarefas de ToM, porém através de uma versão do BH-PTP com alterações expressivas, na qual 75\% dos participantes não registraram o critério para aquisição (domínio) do repertório de relações reversas e duplo-reversas. Montoya-Rodriguez e Molina-Cobos (2016) indicam que resultados da inexistência de efeitos nas tarefas de ToM são consistentes com outros estudos (Jackson et al., 2014; Lovett \& Rehfeldt, 2014), indicando que o BH-PTP pode não ser suficiente para produzir mudanças na tomada de perspectiva mensurada por essas tarefas; mas inconsistentes quando comparados com as evidências de outras investigações que apontam associações entre os repertórios de ToM e MDR (O’Neil \& Weil, 2014; Villatte et al., 2010b; Weil et al., 2011).

De forma geral, as evidências sobre a relação entre as tarefas de ToM e as MDR continuam inconclusivas e apesar da interface do perfil desenvolvimental, ainda não é possível afirmar que os repertórios são relacionados. Porém, vale ressaltar que existe uma consistência entre os estudos que registraram efeitos significativos nas tarefas de ToM, a relação entre o desempenho nas relações reversas e o desempenho nestas tarefas (Hendriks et al., 2016; Villatte et al., 2010b; Weil et al., 2011). Ainda sobre as correlações de outros indicadores com as MDR, Montoya-Rodriguez et al. (2017) indicaram a necessidade de investigar o efeito das MDR em variáveis clínicas. Em nossa revisão, somente Kavanagh 
et al. (2019) examinou possíveis correlações entre diversas escalas psicológicas e os desempenhos no IRAP, não registrando correlações significativas nas análises após as correções.

Os resultados da presente revisão, no geral, sobre as MDR indicam que o treino das relações reversas aparenta possuir maior relevância clínica, pois as relações no nível simples já costumam estar estabelecidas e os repertórios duplo-reversos são muito complexos e não participam de maneira significativa das tarefas em ToM. Referente aos procedimentos de aplicação dos protocolos com os participantes, foi registrada uma tendência geral em direção aos procedimentos vivenciais e interativos (BarberoRubio et al., 2016; Barron et al., 2018; Belisle et al., 2016; Guinther, 2017, 2018; Kavanagh et al., 2018; Montoya-Rodriguez \& Molina-Cobos, 2016, 2019; Montoya-Rodríguez \& McHugh, 2017) e um distanciamento de aplicações envolvendo somente instruções orais ou escritas (Arango \& Baquero, 2017; Hendriks et al., 2016). Tal foco tem por objetivo garantir que repertórios associados à tomada de perspectiva estejam participando das respostas às tentativas e não somente comportamentos de resolução de problema e construção de sentido associados exclusivamente a outros tipos de molduras relacionais.

Somente os artigos de Guinther $(2017,2018)$ não utilizaram o conceito de MDR (McHugh et al., 2004) como fundamentação teórica para a compreensão da tomada de perspectiva e metodológica para a construção do protocolo e das tentativas, limitando a capacidade de articulação entre os resultados obtidos com os resultados dos demais estudos. Para o autor, a propriedade da bidirecionalidade do RRAA não está presente no comportamento dêitico, pois estes são comportamentos de sinalizar (pointing behavior), sendo assim, não existiriam molduras do tipo dêiticas. Entretanto, para Guinther (2017) a tomada de perspectiva ocorre através do RRAA, por meio de respostas relacionais arbitrárias envolvendo relações materiais e espaço-temporais. Respostas derivadas podem ser produzidas quando existe uma triangulação entre as relações Sujeito-Estímulo, Outro-Estímulo, SujeitoOutro; nas palavras do autor: "(...) Eu postulo que em algumas ocasiões quaisquer duas relações em uma tríade envolvem conjuntamente um terceiro." (Guinther, 2017, p. 4; tradução livre).
O RT-PTP consiste em um procedimento composto por tarefas de discriminação com complexidade crescente, baseadas em tentativa e no erro, com um procedimento de matching-to-sample (MTS); as tentativas são apresentadas no computador e são baseadas em tarefas visoespaciais. As tarefas de discriminação, em ordem crescente de complexidade, foram: estabelecer controle contextual para os estímulos arbitrários sobre as perspectivas dos "outros"; estabelecer relações materiais entre a perspectiva do "eu" e o "estímulo 1"; estabelecer relações espaço-temporais entre a perspectiva do "eu" e o "estímulo 2"; estabelecer relações espaço-temporais relativas da perspectiva do "outro" com o "estímulo 2"; estabelecer relações espaço-temporais relativas de proximidade da perspectiva do "outro" com o "estímulo 2"; teste de triangulação da perspectiva do "outro" com o "estímulo 1" (Guinther, 2017).

O autor foi capaz de estabelecer o controle contextual sobre a tomada de perspectiva, por meio da participação do comportamento dêitico de apontar em molduras relacionais de coordenação entre as relações materiais entre si e os outros, derivando crenças falsas (Guinther, 2018) e verdadeiras (Guinther, 2017), através da triangulação relacional (RT). Os resultados registrados pelo o autor são iniciais, porém consistentes com as previsões da RT sobre o processo de derivação de respostas sobre a perspectiva dos outros. Não existem outros artigos publicados com a mesma abordagem, representando uma nova explicação da RFT sobre a tomada de perspectiva, requisitando mais investigações para replicar os resultados, assim como sinalizando a necessidade de expandir as evidências com outras molduras relacionais além das molduras de coordenação, e com populações clínicas com déficits na tomada de perspectiva (Guinther, 2017).

A abordagem RT (Guinther, 2017) surgiu através de problemáticas oriundas do conceito de MDR identificadas pelo autor:

É, portanto, discutível se o MBH-PTP fornece as demonstrações de implicação e transformação de função que são necessárias para evidenciar a influência contextual sobre a moldura relacional, mesmo enquanto o MBH-PTP prevê com sucesso a fluência de tomada de perspectiva. (p. 17; tradução livre) 
A crítica feita por Guinther (2017) levanta o questionamento sobre a existência de implicação entre as relações indicadas pelas MDR, sinalizando que determinada propriedade associada a "Você" não possui implicações mútuas para o "Eu" (assim como para as relações Aqui-Ali, AgoraDepois). Desta forma, sem a implicação mútua, também não é possível apresentar a propriedade de transformação de função de estímulo. Hayes et al. (2001) indicam que para uma relação bidirecional entre os estímulos, se a primeira relação é especificada, a segunda deve ser derivada por implicação mútua. Entretanto, de fato, não está claro de que forma as relações estabelecidas por um estímulo "Aqui" e "Agora", implicam caraterísticas ou funções para os estímulos situados nas relações "Ali" e "Depois" respectivamente. Sem a produção de implicação, mútua e combinatória, e transformação de função de estímulo, não podemos afirmar que molduras relacionais estão em ação, pois essas são propriedades que a caracterizam e tornam possível determinar a natureza da relação e diferenciar os tipos de molduras relacionais (Hayes et al., 2001).

Ao examinar uma relação envolvendo uma moldura relacional Maior/Menor, temos o seguinte formato: "A maior que B, B menor que A. Quem é maior? Quem é menor?". Caso a dica contextual relacional "Maior/Menor" seja substituída por uma sequência qualquer da mesma letra, XXX(maior)/ YYY(menor), por exemplo, ficamos com a seguinte relação: “A XXX B, B YYY A". Verifica-se que a mudança não mais permite a especificação da relação a ser estabelecida: é uma rede relacional incompleta (Hayes, et al., 2001), indicando que a dica contextual fundamental para determinar a natureza da relação entre os estímulos foi afetada. Porém, no caso das MDR, como elaborada por uma tentativa do BH-PTP, temos a seguinte estrutura (moldura dêitica pessoal Você-Eu): "Você está com uma pedra azul. Eu estou com uma pedra branca. Com qual pedra você está? Com qual pedra eu estou?”. Ao realizar o mesmo procedimento, substituindo a dica contextual relacional pessoal (Você = YYY/ Eu = XXX), ficamos com a seguinte estrutura: "XXX está com uma pedra azul, YYY está com uma pedra branca." O sentido da relação permaneceu, uma rede relacional completa, sinalizando que as pala- vras Eu-Você não são as dicas contextuais centrais para determinar a natureza da relação que deve ser aplicada aos estímulos apresentados nesse tipo de afirmação, e portanto, outros tipos de molduras relacionais (não-dêiticas), ou respostas relacionais não-arbitrárias, podem ser as responsáveis pela especificação da natureza da relação entre os estímulos, e consequentemente pela dinâmica da transformação de função de estímulo. Para além desse aspecto, Guinther (2017) também aponta questionamentos sobre a estrutura das tentativas presentes no BH-PTP: “(...) qualquer estímulo poderia substituir os termos do apontamento dêitico e ainda seria de se esperar que o feedback operante melhorasse o desempenho no MBH-PTP com base simplesmente nas formas gerais das perguntas (...)" (p. 17; tradução livre), sugerindo que o formato básico das tentativas do BH-PTP pode estar envolvendo outras formas de derivação de respostas como a generalização de estímulos.

Portanto, em função dessas dificuldades, são necessárias maiores investigações para diferenciar e determinar que são as MDR que estão operando e não outros tipos de RRAA, através da pesquisa sobre a natureza das propriedades de implicação presentes nas MDR. Ainda tratando das propriedades básicas das molduras relacionais, a transformação de função de estímulo é um resultado fundamental para determinar o caráter da moldura relacional envolvida. Os estudos com transformação de função de estímulo das MDR ainda estão restritos à função de estímulo discriminativo, às dicas contextuais "Eu-Você/Aqui-Ali/ Agora-Depois", também contribuindo para as dificuldades nos processos de diferenciação e definição das caraterísticas das MDR, sendo necessárias pesquisas que envolvam transformações de funções de estímulo mais salientes e variadas, como estímulos reforçadores, punidores e eliciadores, presentes em estudos clássicos da RFT (Hayes et al., 1991; Roche et al., 2000). Deste modo, será possível avaliar, por exemplo, as previsões sobre a transformação de funções emocionais através das MDR (Montoya-Rodríguez et al., 2017).

Apesar da afirmação de Hayes et al. (2001) "O número de maneiras pelas quais os estímulos podem ser relacionados é grande, e isso significa que existem muitos tipos específicos de molduras re- 
lacionais.” (p. 35; tradução livre), são necessárias evidências empíricas da produção de um padrão específico de implicação e transformação de função de estímulo, que não podem ser explicadas pelas molduras relacionais já descritas, para justificar a existência de um novo tipo de RRAA, pois: “(...) padrões de transformação ajudam a definir a natureza de uma resposta relacional (...)" (Hayes et al., 2001, p. 62; tradução livre), e a ausência de pesquisas para caracterizar a transformação de função de estímulo nas MDR, para além dos estímulos discriminativos, impossibilitam a identificação desse padrão.

As duas abordagens para a tomada de perspectiva na RFT, as MDR e a triangulação relacional, parecem concordar de que se trata de uma habilidade complexa, constituída de outros repertórios mais simples, que participam de redes de molduras relacionais, apresentando as propriedades de implicação e transformação de função de estímulo. A divergência emerge com mais saliência na explicação de quais são os repertórios básicos que constituem a tomada de perspectiva; exposto de outra maneira, é possível sugerir que a discordância reside na compreensão sobre o conceito de perspectiva. De acordo com a abordagem das MDR (McHugh et al., 2004), elas representam os repertórios básicos, que depois de estabelecidos, vão participar da tomada de perspectiva. Já para a RT (Guinther, 2017), os repertórios básicos se referem ao comportamento dêitico de apontar, não caracterizando um tipo de moldura relacional, mas que pode vir a integrar as molduras relacionais, como as molduras de oposição e de coordenação.

Emergiram algumas questões que potencialmente podem contribuir para explorar a natureza da tomada de perspectiva, estendendo a leitura de Guinther (2017) sobre o fenômeno da tomada de perspectiva. Levou-se em consideração a existência de aspectos não-arbitrários (comportamento de apontar) participando do controle da aplicação das molduras relacionais (tomada de perspectiva), sendo possível conceituar a tomada de perspectiva como um tipo de análise pragmática verbal. Segundo Hayes et al. (2001):

(...) a análise pragmática verbal refere-se ao enquadramento relacional sob o controle de características abstraídas do ambiente não arbitrário que são eles próprios enquadrados relacionalmente. Dito em outras palavras, a análise pragmática verbal envolve agir verbalmente sobre o mundo e, como resultado, fazer com que o mundo desempenhe funções verbais. (p. 90; tradução livre)

Neste sentido, os repertórios mais simples da tomada de perspectiva estariam associados as relações entre aspectos não-arbitrários do ambiente, como as posições espaço-temporais relativas (comportamento dêitico de apontar), que depois de serem abstraídas passam a integrar as redes relacionais. Este processo constituiria as habilidades envolvidas na tomada de perspectiva, sendo esta habilidade uma forma de análise verbal de aspectos não-arbitrários do ambiente. Associado a esse conceito apresentado pela RFT, é possível deduzir que além do comportamento de apontar, o comportamento verbal definido como tato (Skinner, 1957), que apresenta contrapartes verbais e não-verbais (Barnes-Holmes et al., 2000), possui potencial na conceituação da tomada de perspectiva. Perguntas como: "O que você está sentindo?", "O que você está vendo?”, “O que você está segurando?", podem gerar respostas que estão sob o controle de estímulos discriminativos não-verbais e não sob o controle de dicas contextuais abstraídas, portanto, serem respostas nas quais o organismo está emitindo um tato, e não realizando uma RRAA; determinadas propriedades tateadas com frequência, através de um treino de múltiplos exemplares, podem ser abstraídas e posteriormente participarem de molduras relacionais (BarnesHolmes et al., 2000).

Por fim, é importante destacar que a integração de dados obtidos a partir de modelos teóricos e procedimentais distintos limitam o alcance dos resultados da atual revisão. Assim como a participação de somente um pesquisador nas etapas de triagem, extração e categorização abre margem para possíveis vieses nos resultados do trabalho. 


\section{Considerações Finais}

A RFT produziu uma agenda prolifera de pesquisas sobre habilidades e fenômenos clássicos da psicologia, sendo a tomada de perspectiva mais um exemplo. Passados 16 anos da publicação de McHugh et al. (2004), houve uma produção intensa de evidências da aplicação das molduras relacionais na compreensão da tomada de perspectiva. Já foram identificados diferentes níveis de complexidade dessa habilidade, padrões desenvolvimentais, e grupos clínicos com déficits nas molduras dêiticas, com posterior aquisição do repertório através do condicionamento operante.

Podemos afirmar que os protocolos presentes nas pesquisas com repertórios de tomada de perspectiva e RFT apresentaram efetividade e sensibilidade referentes as mudanças comportamentais associadas a habilidade de tomada de perspectiva, e as evidências oriundas do seu treinamento reforçam a compreensão de que se trata de uma habilidade complexa, composta por um conjunto de repertórios distintos, que integram redes relacionais. Todos os protocolos foram capazes de estabelecer os repertórios desejados através do condicionamento operante e produzir respostas derivadas. Porém, as evidências limitadas sobre a transformação de função de estímulo associada aos repertórios de RRAA envolvidos na tomada de perspectiva comprometem o desenvolvimento do campo. As resoluções dos dilemas teórico-conceituais registrados no presente trabalho devem ser balizados pelos resultados produzidos na superação dessa limitação de caráter empírico. Além disso, as evidências inconsistentes sobre os efeitos dos protocolos, mais especificamente das molduras relacionais dêiticas, nas tarefas de teoria da mente também merecem mais investigações para determinar se existem ou não interfaces entre os repertórios envolvidos nessas tarefas de tomada de perspectiva.

\section{Referências}

*Arango, M. I. R., \& Baquero, L. A. Q. (2017). Investigación traslacional en el estudio de marcos deícticos: Reflexiones a partir de un trabajo experimental. Mexican Journal of Behavior Analysis, 1(43), 97-127. https://doi.org/10.5514/ rmac.v43.i1.61078

*Barbero-Rubio, A., López-López, J. C., Luciano, C., \& Eisenbeck, F. (2016). Perspective-taking measured by implicit relational assessment procedure (IRAP). The Psychological Record, 66, 243-252. https://doi.org/10.1007/s40732-0160166-3

Barnes-Holmes, D., Barnes-Holmes, Y., \& Cullinan, V. (2000). Relational frame theory and Skinner's verbal behavior: A possible synthesis. The Behavior Analyst, 23(1), 69-84. https://doi. org/10.1007/BF03392000

*Barron, B. F., Verkuylen, L., Belisle, J., Paliliunas, D., \& Dixon, M. R. (2019). Teaching "then-later" and "here-there" relations to children with autism: An evaluation of single reversals and transformation of stimulus function. Behavior Analysis Practice, 12, 167-175. https://doi. org/10.1007/s40617-018-0216-1

Baron-Cohen, S., Wheelwright, S., Hill, J., Raste, Y., Plumb, I. (2001). The "reading the mind in the eyes" test revised version: A study with normal adults, and adults with asperger syndrome or high-functioning autism. Journal of Child Psychology and Psychiatry, 42(2), 241-251.

*Belisle, J., Dixon, M. R., Stanley, C. R., Munoz, B., \& Daar, J. H. (2016). Teaching foundational perspective-taking skills to children with autism using the PEAK-T curriculum: Singlereversal "I-You" deictic frames. Journal of Applied Behavior Analysis, 49(4), 965-969. https://doi.org/10.1002/jaba.324

Cassidy, S., Roche, B., \& O'Hora, D. (2010). Relational frame theory and human intelligence. European Journal of Behavior Analysis, 11(1), 37-51. https://doi.org/10.1080/15021149 .2010 .11434333

Davlin, N. L., Rehfeldt, A. R., \& Lovett, S. (2011). A relational frame theory approach to understanding perspective-taking using children's sto- 
ries in typically developing children. European Journal of Behavior Analysis, 12(2), 403-430. https://doi.org/10.1080/15021149.2011.11434392

Dixon, M. R., Belisle, J., McKeel, A., Whiting, S., Speelman, R., Daar, J. H., Rowsey, K. (2017). An internal and critical review of the PEAK relational training system for children with autism and related intellectual disabilities: 2014-2017. The Behavior Analyst, 40, 493-521. https://doi. org/10.1007/s40614-017-0119-4

Gilroy, S. P., Lorah, E. R., Dodge, J., \& Fiorello, C. (2015). Establishing deictic repertoires in autism. Research in Autism Spectrum Disorders, 19, 82-92. https://doi.org/10.1016/j. rasd.2015.04.004

Gore, N. J., Barnes-Holmes, Y., \& Murphy, G. (2010). The relationship between intellectual functioning and relational perspective-taking. International Journal of Psychology and Psychological Therapy, 10(1), 1-17.

${ }^{\star}$ Guinther, P. M. (2017). Contextual influence over deriving others' true beliefs using a relational triangulation perspective-taking protocol (RTPTP-M1). Journal of the Experimental Analysis of Behavior, 108(3), 433-456. https://doi. org/10.1002/jeab.291

${ }^{\star}$ Guinther, P. M. (2018). Contextual influence over deriving another's false beliefs using a relational triangulation perspective taking protocol (RT-PTP-M2). Journal of the Experimental Analysis of Behavior, 110(3), 500-521. https:// doi.org/10.1002/jeab.480

Hayes, S. C., Kohlenberg B. S., \& Hayes, L. J. (1991). The transfer of specific and general consequential functions through simple and conditional equivalence relations. Journal of the Experimental Analysis of Behavior, 56(1), 119137. https://doi.org/10.1901/jeab.1991.56-119

Hayes, S. C., Barnes-Holmes, D., \& Roche, B. (Eds.). (2001). Relational frame theory: A post-skinnerian account of human language and cognition. Kluwer Academic.

${ }^{\star}$ Hendriks, A. L., Barnes-Holmes, Y., McEnteggart, C., Mey, H. R. A., Witteman, C. L. M., Janssen, G. T. L., \& Egger, J. I. M. (2016). The relationship between theory of mind and relational frame theory: Convergence of perspective-taking measures. Clinical Neuropsychiatry, 13(2), 17-23.
Jackson, M. L., Mendoza, D. R., \& Adams, A. N. (2014). Teaching a deictic relational repertoire to children with autism. The Psychological Record, 64, 791-802. https://doi.org/10.1007/ s40732-014-0078-z

Janssen, G., Mey, H., Hendriks, A., Koppers, A., Kaarsemaker, M., Witteman, C., \& Egger, J. (2014). Assessing deictic relational responding in individuals with social anxiety disorder: Evidence of perspective-taking difficulties. The Psychological Record, 64, 21-29. https://doi. org/10.1007/s40732-014-0013-3

*Kavanagh, D., Barnes-Holmes, Y., BarnesHolmes, D., McEnteggart, C., \& Finn, M. (2018). Exploring differential trial-type effects and the impact of a read-aloud procedure on deictic relational responding on the IRAP. The Psychological Record, 68, 163-176. https://doi. org/10.1007/s40732-018-0276-1

Kavanagh, D., Barnes-Holmes, Y., \& BarnesHolmes, D. (2020). The study of perspective-taking: Contributions from mainstream psychology and behavior analysis. The Psychological Record, 70, 581-604. https://doi. org/10.1007/s40732-019-00356-3

*Kavanagh, D., Roelandt, A., Raemdonck, L. V., Barnes-Holmes, Y., Barnes-Holmes, D., \& McEnteggart, C. (2019). The on-going search for perspective-taking IRAPs: Exploring the potential of the natural language-IRAP. The Psychological Record, 69, 291-314. https://doi. org/10.1007/s40732-019-00333-w

Lovett, S., \& Rehfeldt, R. A. (2014). An evaluation of multiple exemplar instruction to teach perspective-taking skills to adolescents with asperger syndrome. Behavioral Development Bulletin, 19(2), 22-36. https://doi.org/10.1037/h0100575

McHugh, L., Barnes-Holmes, Y., \& Barnes-Holmes, D. (2004). Perspective-taking as relational responding: A developmental profile. The Psychological Record, 54, 115-144. https://doi. org/10.1007/BF03395465

McHugh. L. (2015). A contextual behavioural science approach to the self and perspective taking. Current Opinion in Psychology, 2, 6-10. https://doi.org/10.1016/j.copsyc.2014.12.030

*Montoya-Rodríguez, M. M., \& Molina-Cobos, F. J. (2016). Relationship between deictic relational 
responding and theory of mind tasks in children: A pilot study. The Psychological Record, 66, 573-587. https://doi.org/10.1007/s40732016-0193-0

*Montoya-Rodríguez, M. M., \& McHugh, L. (2017). Teaching perspective-taking skills to an adult with down syndrome: A case study. Journal of Contextual Behavioral Science, 6(3), 293-297. https://doi.org/10.1016/j.jcbs.2017.04.012

Montoya-Rodríguez, M. M., Molina, F. J., \& McHugh, L. (2017). A review of relational frame theory research into deictic relational responding. The Psychological Record, 67, 569-579. https://doi.org/10.1007/s40732-016-0216-X

*Montoya-Rodríguez, M. M., \& Molina-Cobos, F. J. (2019). Training perspective taking skills in individuals with intellectual disabilities: A functional approach. Journal of Contextual Behavioral Science, 14, 1-10. https://doi. org/10.1016/j.jcbs.2019.08.003

O’Neill, J., \& Weil, T. M. (2014). Training deictic relational responding in people diagnosed with schizophrenia. The Psychological Record, 64, 301-310. https://doi.org/10.1007/s40732-0140005-3

Rehfeldt, R. A., Dillen, J. E., Ziomek, M. M., \& Kowalchuk, R. K. (2007). Assessing relational learning deficits in perspective-taking in children with high-functioning autism spectrum disorder. The Psychological Record, 57, 23-47. https://doi.org/10.1007/BF03395563

Rehfeldt, R. A., Ming, S., \& Belisle, J. (2020). Relational Framing. In Stephen Hupp, S., \& Jewell, J. D. (Eds.), The Encyclopedia of Child and Adolescent Development. John Wiley \& Sons, Inc. https://doi.org/10.1002/9781119171492. wecad066

Roche, B., Barnes-Holmes, D., Barnes-Holmes, Y., Smeets, P. M., \& Mcgeady, S. (2000). Contextual control over the derived transformation of discriminative and sexual arousal functions. The Psychological Record, 50, 267-291. https://doi. org/10.1007/BF03395356

Rose, J. C. C., \& Rabelo., L. Z. (2012). Teoria das molduras relacionais e possíveis aplicações à educação. Revista Di, 3. 10-15.

Skinner, B. F. (1957) Verbal Behavior. B. F. Skinner Foundation.
Souza, M. T., Silva, M. D., \& Carvalho, R. (2010). Revisão integrativa: O que é e como fazer. Einstein (São Paulo), 8(1), 102-106. https://doi. org/10.1590/s1679-45082010rw1134

Vilardaga, R., Estévez, A., Levin, M. E., \& Hayes, S. C. (2012). Deictic relational responding, empathy, and experiential avoidance as predictors of social anhedonia: Further contributions from relational frame theory. The Psychological Record, 62, 409-432. https://doi.org/10.1007/ BF03395811

Villatte, M., Monestès, J., McHugh, L., Baquéa, E. F., \& Loas, G. (2008). Assessing deictic relational responding in social anhedonia: A functional approach to the development of theory of mind impairments. International Journal of Behavioral Consultation and Therapy, 4(4), 360-373.

Villatte, M., Monestès, J., McHugh, L., Baquéa, E. F., \& Loas, G. (2010a). Assessing perspective taking in schizophrenia using relational frame theory. The Psychological Record, 60, 413-436. https://doi.org/10.1007/BF03395719

Villatte, M., Monestès, J., McHugh, L., Baquéa, E. F., \& Loas, G. (2010b). Adopting the perspective of another in belief attribution: Contribution of relational frame theory to the understanding of impairments in schizophrenia. Journal of Behavior Therapy and Experimental Psychiatry, 41(2), 125-134. https://doi.org/10.1016/j.jbtep.2009.11.004

Weil, T. M., Hayes, S. C., \& Capurro, P. (2011). Establishing a deictic relational repertoire in young children. The Psychological Record, 61, 371-390. https://doi.org/10.1007/BF03395767

\section{Informações do Artigo}

Histórico do artigo:

Submetido em: 28/11/2020

Primeira decisão editorial: 16/04/2021

Aceito em: 12/05/2021

Editor: William F. Perez 